TITLE:

\title{
State-specific solvation effect on the intramolecular charge transfer reaction in solution: A linear- response free energy TDDFT method
}

\author{
AUTHOR(S): \\ Minezawa, Noriyuki
}

CITATION:

Minezawa, Noriyuki. State-specific solvation effect on the intramolecular charge transfer reaction in solution: A linear-response free energy TDDFT method. Chemical Physics Letters 2014, 608: 140-144

ISSUE DATE:

2014-07-21

URL:

http://hdl.handle.net/2433/189097

RIGHT:

(c) 2014 Elsevier B.V.; この論文は出版社版でありません。引用の際には 出版社版をご確認ご利用ください。; This is not the published version. Please cite only the published version. 


\title{
State-specific solvation effect on the intramolecular charge transfer reaction in solution: A linear-response free energy TDDFT method
}

\author{
Noriyuki Minezawa \\ Fukui Institute for Fundamental Chemistry, Kyoto University, \\ Sakyo-ku, Kyoto 606-8103, Japan \\ E-mail: minezawa@fukui.kyoto-u.ac.jp
}

Tel: +81-75-711-7902

\begin{abstract}
State-specific solvation for constructing excited-state free energy surfaces in solution is discussed within the framework of reference interaction site model/time-dependent density functional theory. The self-consistency between the solute electron density and solvation structure is achieved using the linear-response free energy approach. The proposed method is applied to the intramolecular charge-transfer (ICT) state formation of 4-(N-pyrrolo)benzonitrile. The linear-response solvation method underestimates the ICT state severely, and the calculation predicts erroneously that the reaction is endergonic. Therefore, it is essential to apply the state-specific method when the transition density fails in describing the solute-solvent interaction upon excitation.
\end{abstract}




\section{Keywords}

Reference interaction site model self-consistent field (RISM-SCF); electronically excited states; twisted intramolecular charge transfer (TICT); 4-(N-pyrrolo)benzonitrile

\section{Introduction}

Constructing equilibrium free energy surfaces for electronically excited states is a first step toward understanding the photochemical processes of molecules in solution. The solute electron density and solvent polarization needs to be self-consistent in order to obtain the equilibrium solvation for excited states. However, it is a difficult task to achieve the self-consistency for the linear-response time-dependent density functional theory (LR-TDDFT) [1-3], because the iterative scheme for correlated methods may be inconsistent due to the involvement of higher-order corrections [4]. Although the discussion in Ref. [4] is based on the second-order Møller-Presset perturbation theory (MP2), the result is transferable to the TDDFT when the

Hartree-Fock reference, MP2 density, and second-order correlation energy are replaced with the DFT reference, TDDFT excited-state density, and excitation energy, respectively. 
The self-consistency between the solute electron density and solvent electronic polarization has been examined in the computation of absorption spectra. In the polarizable continuum model (PCM), the state-specific (SS) and LR formulations differ significantly [5-8]. The former considers the solute-solvent interaction explicitly using the difference between the ground-state and excited-state expectation values, while the latter describes the corresponding energy as the direct product of transition density. The effect of SS solvation has been discussed in the framework of equation-of-motion coupled cluster/polarizable solvent model [9-11]. The selection of solvation models has influence on the energy profile as well as the excitation energy. Pedone has recently examined the energy profile of coumarin dye in polar solvents at the TDDFT-PCM level and pointed out the failure in the LR solvation model [12].

The author has recently introduced an efficient method of constructing excited-state free energy surfaces in solution [13]. This method, LR-RISM-TDDFT or simply "RISM-TDDFT", combines the LR-TDDFT with the reference interaction site model self-consistent field (RISM-SCF) method [14-20]. The developed approach, however, assumes the LR solvation; the excited-state free energy is given as the sum of the ground-state equilibrium free energy and the relevant 
excitation energy given as a pole of reference electronic structure. In other words, this formulation lacks the self-consistency between the excited-state solute density and solvent polarization. The hybrid method of LR-TDDFT and RISM-SCF has been developed in other groups [21-23]. Recently, Hayaki et al. [23] has constructed the excited-state non-equilibrium free energy surfaces, which obtained by solving the RISM equation under hypothetical charge distribution that connects the ground and excited states.

In this Letter, the self-consistency problem is considered within the framework of RISM integral equation theory/LR-TDDFT. The excited-state equilibrium free energy is given using the linear-response free energy (LRFE) method [24-28], in which the self-consistency between the solute electron density and solvent polarization is achieved by solving the linear equation. The method is applied to the intramolecular charge transfer (ICT) state formation of 4-(N-pyrrolo)benzonitrile (PBN). This molecular system is a good example because the solute electron density of ICT state differs significantly from that of the reference ground state. Therefore, the excited-state free energy depends critically on the solvent reorganization.

\section{LRFE-TDDFT method}




\subsection{Equilibrium free energy for excited states}

In the present work, a hybrid method of LR-TDDFT and LRFE is developed (hereafter denoted as "LRFE-TDDFT"). The equilibrium free energy for electronically excited states is determined by minimizing the following non-equilibrium free energy [24-28]

$$
\mathcal{G}_{\text {noneq }}(\mathbf{R}, \mathbf{V})=E_{\text {elec }}(\mathbf{R}, \mathbf{V})-\mathbf{V}^{T} \mathbf{Q}_{0}+\Delta \mu_{0}+\frac{1}{2 \beta}\left(\mathbf{V}-\mathbf{V}_{0}\right)^{T} \mathbf{C}_{0}^{-1}\left(\mathbf{V}-\mathbf{V}_{0}\right)
$$

The first term describes the solute electronic energy under the influence of external electrostatic potential (ESP) V. $\mathbf{Q}_{0}$ and $\Delta \mu_{0}$ are the partial charges and excess chemical potential for the reference state, respectively, and are determined by solving the ground-state RISM-DFT. The last term describes the solvent fluctuation around the reference-state solvation structure. The quadratic form means that the probability of finding the solvation coordinate $\mathbf{V}$ around the reference-state ESP $\mathbf{V}_{0}$ is given by the multi-dimensional Gaussian distribution $[29,30] . \quad \beta$ is the inverse temperature, and the matrix $\mathbf{C}_{0}$ describes the ESP covariance

$$
\mathbf{C}_{0} \equiv\left\langle\Delta \mathbf{V} \Delta \mathbf{V}^{T}\right\rangle_{0}=-\beta^{-1} \frac{\partial \Delta \mu_{0}}{\partial \mathbf{Q}_{0} \partial \mathbf{Q}_{0}}=-\beta^{-1} \frac{\partial \mathbf{V}_{0}}{\partial \mathbf{Q}_{0}}
$$

which is evaluated solving the first-order coupled-perturbed RISM (CP-RISM) equations [30].

The solute electronic energy under the ESP $\mathbf{V}$, the first term of Eq. (1), is given by the sum of 
ground-state Kohn-Sham energy functional $E_{\mathrm{KS}}(\mathbf{R}, \mathbf{V})$ [31] and the excitation energy $\Omega(\mathbf{R}, \mathbf{V})$,

$$
E_{\text {elec }}(\mathbf{R}, \mathbf{V})=E_{\mathrm{KS}}(\mathbf{R}, \mathbf{V})+\Omega(\mathbf{R}, \mathbf{V})
$$

To take into account the influence of external ESP, $E_{\mathrm{KS}}(\mathbf{R}, \mathbf{V})$ is computed using the so-called solvated Fock matrix [14,15],

$$
\hat{F}^{\text {solv }}=\hat{F}^{\mathrm{KS}}+\mathbf{V}^{T} \hat{\mathbf{Q}}
$$

Here $\hat{F}^{\mathrm{KS}}$ is the usual gas-phase Kohn-Sham Fock operator, and $\hat{\mathbf{Q}}$ is charge generation operator. The excitation energy $\Omega$ and transition amplitudes $\mathbf{X}$ and $\mathbf{Y}$ are determined by solving the LR-TDDFT equation [2]

$$
\left(\begin{array}{ll}
\mathbf{A} & \mathbf{B} \\
\mathbf{B} & \mathbf{A}
\end{array}\right)\left(\begin{array}{l}
\mathbf{X} \\
\mathbf{Y}
\end{array}\right)=\Omega(\mathbf{V})\left(\begin{array}{cc}
\mathbf{1} & \mathbf{0} \\
\mathbf{0} & -\mathbf{1}
\end{array}\right)\left(\begin{array}{l}
\mathbf{X} \\
\mathbf{Y}
\end{array}\right)
$$

The coupling matrices $\mathbf{A}$ and $\mathbf{B}$ are not modified at all, because the ESP $\mathbf{V}$ is thought of as the external potential. The excitation energy $\Omega(\mathbf{V})$, however, depends on $\mathbf{V}$ through molecular orbital (MO) coefficients and orbital energies.

The equilibrium solvation structure for excited states is given as the extremum of non-equilibrium free energy, Eq. (1). By taking the derivative with respect to the ESP,

$$
\begin{aligned}
\frac{\partial \mathcal{G}_{\text {noneq }}}{\partial \mathbf{V}} & =\frac{\partial E_{\text {elec }}}{\partial \mathbf{V}}-\mathbf{Q}_{0}+\frac{1}{\beta} \mathbf{C}_{0}^{-1}\left(\mathbf{V}-\mathbf{V}_{0}\right) \\
& \equiv \tilde{\mathbf{Q}}-\mathbf{Q}_{0}+\frac{1}{\beta} \mathbf{C}_{0}^{-1}\left(\mathbf{V}-\mathbf{V}_{0}\right)
\end{aligned}
$$


$\mathbf{Q}$ is the derivative of $E_{\text {elec }}$ in Eq. (3) with respect to $\mathbf{V}$ and has a dimension of charge. In the present LR-TDDFT case, $\mathbf{Q}$ is obtained by solving the CP-KS equation [3] and given as $\operatorname{Tr}[(\mathbf{D}+\mathbf{P}) \hat{\mathbf{Q}}]$, where $\mathbf{D}$ and $\mathbf{P}$ are the ground-state density matrix and relaxed difference density matrix, respectively. Since the equilibrium solvation coordinate (ESP) is given as the extremum of non-equilibrium free energy, the second line of Eq. (6) is set equal to zero. The resultant ESP is obtained by

$$
\mathbf{V}=\mathbf{V}_{0}-\beta \mathbf{C}_{0}\left(\tilde{\mathbf{Q}}-\mathbf{Q}_{0}\right)
$$

Because the ESP is dependent on the site charges $\tilde{\mathbf{Q}}$, Eqs. (3) and (7) are solved in an iterative manner. After the convergence is achieved, the optimal ESP $\mathbf{V}_{\mathrm{eq}}$ is obtained. The equilibrium free energy is given by inserting $\mathbf{V}_{\mathrm{eq}}$ into Eq. (1)

$$
\begin{aligned}
\mathcal{G}^{\mathrm{LRFE}}(\mathbf{R}) & \equiv \mathcal{G}_{\text {noneq }}\left(\mathbf{R}, \mathbf{V}_{\text {eq }}\right) \\
& =E_{\text {elec }}\left(\mathbf{R}, \mathbf{V}_{\text {eq }}\right)-\mathbf{V}_{\text {eq }}^{T} \mathbf{Q}_{0}+\Delta \mu_{0}+\frac{1}{2 \beta}\left(\mathbf{V}_{\text {eq }}-\mathbf{V}_{0}\right)^{T} \mathbf{C}_{0}^{-1}\left(\mathbf{V}_{\text {eq }}-\mathbf{V}_{0}\right)
\end{aligned}
$$

The analytic gradient of Eq. (8) is computed as described in Ref. [27].

\subsection{Comparison with RISM-TDDFT}

Here the LRFE-TDDFT method is compared to the previous RISM-TDDFT [13]. As derived in 
the Appendix, the equilibrium free energy in Eq. (8) is expanded around the ground-state reference ESP $\mathbf{V}_{0}$,

$$
\mathcal{G}^{\mathrm{LRFE}}=\mathcal{G}_{0}+\Omega\left(\mathbf{V}_{0}\right)+\Delta \mathbf{V}^{T} \Delta \mathbf{Q}^{\mathrm{LRFE}}+\frac{1}{2} \Delta \mathbf{V}^{T}\left(\mathbf{K}+\beta^{-1} \mathbf{C}_{0}^{-1}\right) \Delta \mathbf{V}
$$

where $\Delta \mathbf{V}=\mathbf{V}_{\text {eq }}-\mathbf{V}_{0}$ and $\Delta \mathbf{Q}^{\mathrm{LRFE}} \equiv(\partial \Omega / \partial \mathbf{V})$. The first term is the ground-state RISM-DFT free energy [14]

$$
\mathcal{G}_{0} \equiv E_{\mathrm{KS}}\left(\mathbf{V}_{0}\right)-\mathbf{V}_{0}^{T} \mathbf{Q}_{0}+\Delta \mu_{0}
$$

The matrix $\mathbf{K}$ in the last term is the charge-response kernel: $\mathbf{K} \equiv \partial \tilde{\mathbf{Q}} / \partial \mathbf{V}$ [32]. By combining Eqs. (7) and (9), the LRFE free energy is

$$
\mathcal{G}^{\mathrm{LRFE}}=\mathcal{G}_{0}+\Omega\left(\mathbf{V}_{0}\right)+\frac{1}{2} \Delta \mathbf{V}^{T} \Delta \mathbf{Q}^{\mathrm{LRFE}}
$$

as derived in the Appendix.

In the previous study [13], the RISM-TDDFT free energy is derived as follows:

$$
\mathcal{G}^{\mathrm{RISM}}=\mathcal{G}_{0}+\Omega^{\mathrm{RISM}}
$$

In the computation of excitation energy $\Omega^{\mathrm{RISM}}$, the LR-TDDFT coupling matrices include the RISM kernel $f^{\text {RISM }}$ that partially accounts for the solvent relaxation in the electronically excited state [13]. Since both $\Omega\left(\mathbf{V}_{0}\right)$ and $\Omega^{\mathrm{RISM}}$ are obtained using the same reference state, $\Omega^{\mathrm{RISM}}$ can be approximated as follows: 


$$
\begin{aligned}
\Omega^{\mathrm{RISM}}\left(\mathbf{V}_{0}\right) & \simeq \Omega\left(\mathbf{V}_{0}\right)+(\mathbf{X}+\mathbf{Y})^{T} \mathbf{f}^{\mathrm{RISM}}(\mathbf{X}+\mathbf{Y}) \\
& =\Omega\left(\mathbf{V}_{0}\right)+\left(\mathbf{Q}^{X+Y}\right)^{T}\left(\frac{\partial \mathbf{V}_{0}}{\partial \mathbf{Q}_{0}}\right)\left(\mathbf{Q}^{X+Y}\right)
\end{aligned}
$$

where $\mathbf{Q}^{X+Y}=\operatorname{Tr}[\hat{\mathbf{Q}}(\mathbf{X}+\mathbf{Y})]$. By combining Eqs. (12) and (13), the RISM-TDDFT free energy leads to

$$
\mathcal{G}^{\mathrm{RISM}}=\mathcal{G}_{0}+\Omega\left(\mathbf{V}_{0}\right)+\left(\mathbf{Q}^{X+Y}\right)^{T}\left(\frac{\partial \mathbf{V}_{0}}{\partial \mathbf{Q}_{0}}\right)\left(\mathbf{Q}^{X+Y}\right)
$$

The difference between Eqs. (9) and (14) is easily understood as in the PCM study [6]. The second term is the excitation energy evaluated at the frozen ESP $\mathbf{V}_{0}$. The difference lies in the last term, which accounts for the change in solute-solvent electrostatic interaction upon excitation. In the RISM-TDDFT, the interaction is given by the product of transition-density charge $\mathbf{Q}^{X+Y}$ and transition-density-induced ESP change $\left(\partial \mathbf{V}_{0} / \partial \mathbf{Q}_{0}\right) \mathbf{Q}^{X+Y}$. Contrastively, the ESP and site charges are explicitly evaluated using the excited-state density for the LRFE-TDDFT in Eq. (9). The site charges reflect the excited-state solvation structure and vice versa. Such a feedback effect is missing in the LR approximation.

In the previous study, the corrected LR (cLR) scheme is also proposed for the RISM-TDDFT free energy: 


$$
\mathcal{G}^{\mathrm{cLR}}=\mathcal{G}_{0}+\Omega\left(\mathbf{V}_{0}\right)+\frac{1}{2}\left(\Delta \mathbf{Q}_{0}^{\mathrm{RISM}}\right)^{T}\left(\frac{\partial \mathbf{V}_{0}}{\partial \mathbf{Q}_{0}}\right) \Delta \mathbf{Q}_{0}^{\mathrm{RISM}}
$$

where $\Delta \mathbf{Q}_{0}^{\text {RISM }}=\operatorname{Tr}[\mathbf{P Q} \mathbf{Q}]$ is the partial charge obtained by solving RISM-TDDFT and subsequent CPKS equation. The cLR method assumes the ESP deviation $\Delta \mathbf{V}$ can be described by the first-order Taylor expansion ("ESP-1 scheme" described in Ref. [13]): $\left(\partial \mathbf{V}_{0} / \partial \mathbf{Q}_{0}\right) \Delta \mathbf{Q}_{0}^{\mathrm{RISM}}$

\section{ICT state formation of PBN}

PBN illustrated in Figure 1 is one of the prototypical donor-acceptor systems. The twisted-ICT (TICT) state, where the donor is perpendicular to the acceptor group, has been suggested as the structural changes accompanying the ICT reaction [33]. For PBN, the highest occupied MO (HOMO) is localized on the pyrrole moiety, and the lowest unoccupied MO (LUMO) on the benzonitrile group. The 1B state is described as the HOMO (a) to LUMO (b) excitation and has a strong charge-transfer character. The absorption and emission spectra are measured in both nonpolar and polar solvents [34-36], and the DFT/multi-reference configuration interaction calculation is reported [37]. 


\subsection{Computational Details}

The LRFE-TDDFT method is applied to the ICT state formation of PBN in an acetonitrile solution. The developed codes were interfaced with the program package GAMESS $[38,39]$. The site-site RISM equations were solved with the hyper-netted-chain closure relation. A three-site model by Jorgensen and Briggs [40] was adopted for acetonitrile solvent. In all calculations, density and temperature were set to be $0.777 \mathrm{~g} / \mathrm{cm}^{3}$ and $298.15 \mathrm{~K}$, respectively. The solute Lennard-Jones (LJ) parameters were taken from the AMBER force field [41], and the standard combination rule was applied to compute the site-site LJ interaction potential.

To study the ICT state formation of PBN, the long-range corrected (LC) BLYP functional [42-45] was employed to avoid the severe underestimation of charge-transfer excitation. A range-separation parameter was tuned to be $0.249 \mathrm{bohr}^{-1}$ by using the procedure in Ref. [46]. The basis set employed was Dunning-Hay double zeta plus polarization (double zeta) quality for heavy (hydrogen) atoms. A set of diffuse p functions with the exponents of 0.034 (C) and 0.048 (N) was added to each atom [47]. The adiabatic free energy curves for the $1 \mathrm{~B}$ state were 
constructed imposing the $C_{2}$ symmetry. The torsion angle between the pyrrole and benzonitrile planes was chosen as the reaction coordinate $\tau$. For a given value of $\tau$, the geometry was determined by optimizing the remaining degrees of freedom.

\subsection{Absorption and emission spectra}

Table 1 shows the vertical transition energies of PBN in the gas and solution phases. At the ground-state minimum, the molecule is partially twisted due to the steric repulsion between the pyrrole and benzonitrile moieties: $28.4^{\circ}$ (gas) and $22.6^{\circ}$ (acetonitrile). The $1 \mathrm{~B}$ and $2 \mathrm{~A}$ states are very close to each other at the Franck-Condon (FC) point. The $2 \mathrm{~A}$ state is bright, and the excitation energy is computed to be 4.72 (4.68) $\mathrm{eV}$ in the gas (solution) phase. The vertical excitation energy is minimally dependent on the presence of polar solvent for all three states shown in Table 1.

In contrast, the fluorescence spectrum differs significantly. Note that the ground-state non-equilibrium free energy is also given by Eq. (1), but now the first term, solute electronic energy, is calculated by the DFT, not TDDFT. Because the same electrostatic potential is 
employed for the initial and final states, the second, third, and fourth terms in Eq. (1) cancel out. The emission energy is computed at the TICT geometry, because this structure is the minimum energy point even in the gas phase. A large solvatochromic shift is obtained; the emission energy is computed to be $3.22(2.41) \mathrm{eV}$ in vacuum (acetonitrile). These values are comparable to those measured by experiment: 3.56 and $2.57 \mathrm{eV}$ in $n$-hexane and acetonitrile, respectively [36]. The large shift is attributed to the strong destabilization of donor pyrrole group in polar acetonitrile solvent: HOMO and LUMO energies are $-0.29(-0.25)$ a.u. and $-0.04(-0.04)$ a.u. in vacuum (acetonitrile).

\subsection{Free energy profiles}

Figure $2 \mathrm{a}$ shows the 1B-state energy profiles along the twisting coordinate. The gas-phase potential energy decreases monotonically as the twisting angle increases, and the perpendicular conformation is the minimum energy point. Therefore, the TICT state formation occurs spontaneously and is responsible for the fluorescence spectrum in the gas phase.

In polar acetonitrile solution, there are qualitative differences between the LRFE-TDDFT and 
RISM-TDDFT free energy curves. The RISM free energy without correction is higher in energy than the LRFE-TDDFT for all geometries. Notably, the former increases monotonically from $4.36\left(\tau=0^{\circ}\right)$ to $4.48\left(\tau=90^{\circ}\right) \mathrm{eV}$. At the TICT state, the RISM-TDDFT overestimates the free energy by $0.70 \mathrm{eV}$ compared to the LRFE. It is difficult to validate the RISM free-energy profile because the gas-phase potential energy decreases without any solvent stabilization. The molecule leads to the TICT state spontaneously by the intramolecular twisting motion only. Furthermore, the twisting motion enhances the dipole moment, and the largest solute-solvent interaction is expected at the twisted conformation. Figure $2 b$ shows why the RISM-TDDFT free energy increases along the twisting angle. The RISM-TDDFT equilibrium free energy is plotted along with the FC non-equilibrium free energy, $\mathcal{G}_{0}+\Omega\left(\mathbf{V}_{0}\right)$. At the TICT state the energy difference is minimal, i.e., the solvent relaxation is very small. The negligible solvent polarization is incompatible with the large solute electronic polarization (the dipole moment of 21 and $3 \mathrm{D}$ for the $1 \mathrm{~B}$ and ground states, respectively). Therefore, the RISM-TDDFT provides incorrect free energy profile and predicts erroneously that the ICT state formation of PBN is endergonic. This is because the RISM-TDDFT without correction cannot take accounts of the solvent reorganization properly (see below). 
Contrastively, the RISM-TDDFT curve becomes nearly identical with the LRFE-TDDFT curve when the correction scheme (cLR) is applied. Both the LRFE and cLR free energies decrease along the twisting motion, although the latter slightly exaggerates the free energy change. The comparison among the three methods highlights the importance of taking accounts of excited-state solvation explicitly. The correction scheme (cLR) is a useful alternative to avoid the lack of self-consistency with modest computational costs, because this method requires only the ground-state RISM-DFT followed by single-point TDDFT energy calculation.

Figure 3 shows the deviation of the excited-state free energy $\mathcal{G}$ from the FC free energy $\mathcal{G}_{0}+\Omega\left(\mathbf{V}_{0}\right)$; see Eqs. (8), (12), and (15) for the LRFE-TDDFT, RISM-TDDFT, and cLR free energies, respectively. As discussed above, the deviation is negligible for the RISM-TDDFT, and the curve stays around zero for all twisting angles. At the TICT state, the residual is estimated to be $7 \times 10^{-4} \mathrm{eV}$, and thus the RISM-TDDFT method cannot take accounts of the solvent relaxation in spite of drastic change in the solute electronic structure. This is attributed to the LR solvation model that is inherent in the RISM-TDDFT method. For the TICT state, the transition-density charge $\mathbf{Q}^{X+Y}$ diminishes due to the small overlap between the HOMO and LUMO (the lambda diagnostic value [48] is 0.15$)$. In contrast, a large relaxation $(0.5-0.7 \mathrm{eV})$ is observed for both the 
LRFE-TDDFT and cLR methods. Furthermore, the contribution increases along the twisting coordinate, and this trend is consistent with the strong solute-solvent interaction for the TICT state. Therefore, it is crucial to evaluate the difference site charges $\Delta \mathbf{Q}$ explicitly using the excited-state density when the transition density fails in describing the solute-solvent interaction upon photo-excitation.

Finally, two criteria are proposed to identify the breakdown of LR solvation model. The failure is due to (a) the negligible contribution of transition density, the third term of Eq. (14), and (b) the drastic change in solute electronic structure upon excitation. For point (a), the lambda diagnosis [48] is the method of choice. There is some correlation between the lambda values and the energy deviation: the former (latter) decreases (increases) along the twisting coordinate. This is because the transition charge $\mathbf{Q}^{X+Y}=\operatorname{Tr}[\hat{\mathbf{Q}}(\mathbf{X}+\mathbf{Y})]$ depends on the overlap between the occupied and virtual MOs. For point (b), the correction given in the third term of Eq. (15) estimates how large the solvent relaxation is. Typical values for ionic $1 b$ state are $0.4-0.8 \mathrm{eV}$ while those for less polar $2 a$ state $\sim 0.02 \mathrm{eV}$. The similar trend is observed in the previous study: the derivation is relatively small when the values are less than $0.1 \mathrm{eV}[13]$. 


\section{Summary}

In the present work, the LRFE-TDDFT method is employed to construct excited-state free energy surfaces in solution. The method is based on the state-specific solvation and affords the self-consistency between the solute electron density and solvation structure for the electronically excited state. The proposed method is applied to study the ICT state formation of PBN to examine how the solute-solvent mutual polarization affects the free energy profile. The free energy obtained by the LRFE-TDDFT is qualitatively different from that by the RISM-TDDFT based on the linear-response solvation. The latter underestimates the TICT state severely and provides incorrect free energy profile. As the result, the RISM-TDDFT predicts erroneously that the TICT state formation is endergonic while the LRFE-TDDFT indicates the exergonic reaction. The RISM-TDDFT method is shown to be problematic when the transition-density-based charges are nearly zero due to the small spatial overlap between the occupied and virtual orbitals. The ICT state formation discussed in this work is typical example. The LRFE-TDDFT method computes the solute-solvent interaction explicitly using the difference electron density between the ground and excited states. Therefore, the present LRFE-TDDFT is a promising approach to 
construct the excited-state free energy surfaces even if the transition density fails in describing the solute-solvent interaction change upon photo-excitation.

\section{Appendix A. Derivation of Eqs. (9) and (11)}

Equation (8) is expanded around the reference ESP $\mathbf{V}_{0}$ up to the second order,

$$
\begin{aligned}
\mathcal{G}^{\mathrm{LRFE}} & =E_{\mathrm{KS}}\left(\mathbf{V}_{0}\right)+\Omega\left(\mathbf{V}_{0}\right)+\left[\frac{\partial\left(E_{\mathrm{KS}}+\Omega\right)}{\partial \mathbf{V}}\right]_{\mathbf{V}_{0}} \Delta \mathbf{V}+\frac{1}{2}(\Delta \mathbf{V})^{T}\left(\frac{\partial^{2} E_{\text {solute }}}{\partial \mathbf{V} \partial \mathbf{V}^{\prime}}\right)_{\mathbf{V}_{0}} \Delta \mathbf{V} \\
& -\left(\mathbf{V}_{0}+\Delta \mathbf{V}\right)^{T} \mathbf{Q}_{0}+\Delta \mu_{0}+\frac{1}{2 \beta}(\Delta \mathbf{V})^{T} \mathbf{C}_{0}^{-1}(\Delta \mathbf{V})
\end{aligned}
$$

where Eq. (3) is employed and $\Delta \mathbf{V}=\mathbf{V}_{\text {eq }}-\mathbf{V}_{0}$. By using the relations $\partial E_{\mathrm{KS}} / \partial \mathbf{V}=\mathbf{Q}_{0}$ and $\partial \Omega / \partial \mathbf{V}=\Delta \mathbf{Q}^{\mathrm{LRFE}}$, Eq. (A1) is

$$
\begin{aligned}
\mathcal{G}^{\mathrm{LRFE}}= & {\left[E_{\mathrm{KS}}\left(\mathbf{V}_{0}\right)-\left(\mathbf{V}_{0}\right)^{T} \mathbf{Q}_{0}+\Delta \mu_{0}\right]+\Omega\left(\mathbf{V}_{0}\right)+\Delta \mathbf{Q}^{\mathrm{LRFE}} \Delta \mathbf{V} } \\
& +\frac{1}{2}(\Delta \mathbf{V})^{T}\left[\frac{\partial^{2}\left(E_{\mathrm{KS}}+\Omega\right)}{\partial \mathbf{V} \partial \mathbf{V}^{\prime}}\right]_{\mathbf{V}_{0}} \Delta \mathbf{V}+\frac{1}{2 \beta}(\Delta \mathbf{V})^{T} \mathbf{C}_{0}^{-1}(\Delta \mathbf{V})
\end{aligned}
$$

The three terms in the square bracket is the ground-state free energy in Eq. (10), and the bracket in the sixth term is the CRK: $\mathbf{K} \equiv \partial \tilde{\mathbf{Q}} / \partial \mathbf{V}=\partial^{2} E_{\text {solute }} / \partial \mathbf{V} \partial \mathbf{V}^{\prime}$ 
From Eq. (7), the ESP difference is given by

$$
\begin{aligned}
\Delta \mathbf{V} & \equiv \mathbf{V}_{\text {eq }}-\mathbf{V}_{0} \\
& =-\beta \mathbf{C}_{0}\left[\tilde{\mathbf{Q}}\left(\mathbf{V}_{0}\right)+\left(\frac{\partial \tilde{\mathbf{Q}}}{\partial \mathbf{V}}\right)_{\mathbf{V}_{0}} \Delta \mathbf{V}-\mathbf{Q}_{0}\right] \\
& =-\beta \mathbf{C}_{0}\left(\Delta \mathbf{Q}^{\mathrm{LRFE}}+\mathbf{K} \Delta \mathbf{V}\right)
\end{aligned}
$$

Inserting Eq. (A3) into Eq. (9) leads to Eq. (11). 


\section{Figure captions}

Figure 1. HOMO (left) and LUMO (right) of 4-(N-pyrrolo)benzonitrile (PBN): carbon (cyan), nitrogen (blue), and hydrogen (white) atoms.

Figure 2. (a) Free energy (potential energy) curves of PBN along the twisting coordinate. Dipole moments at $\tau=0^{\circ}$ and $90^{\circ}$ are also shown. (b) Comparison of the RISM-TDDFT and Franck-Condon free energies.

Figure 3. Solvent relaxation energy along the twisting coordinate. 

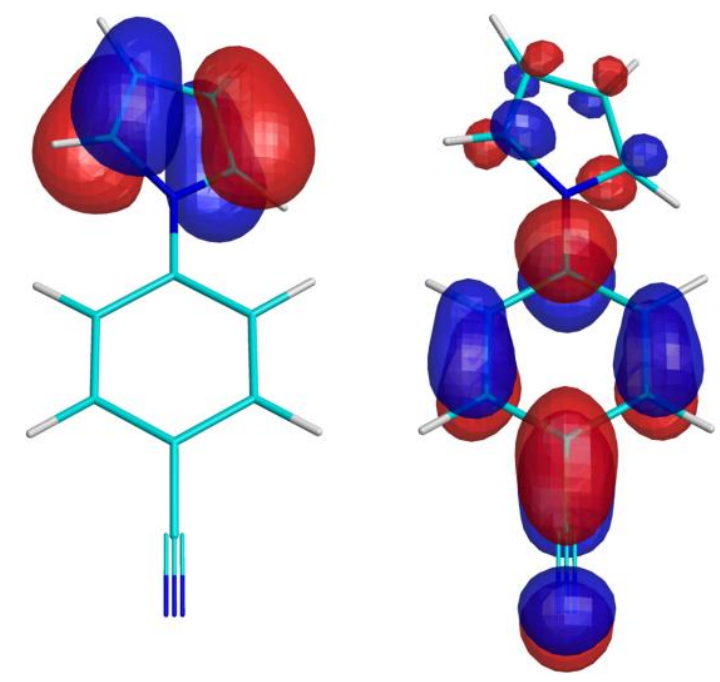

Fig. 1. N. Minezawa 

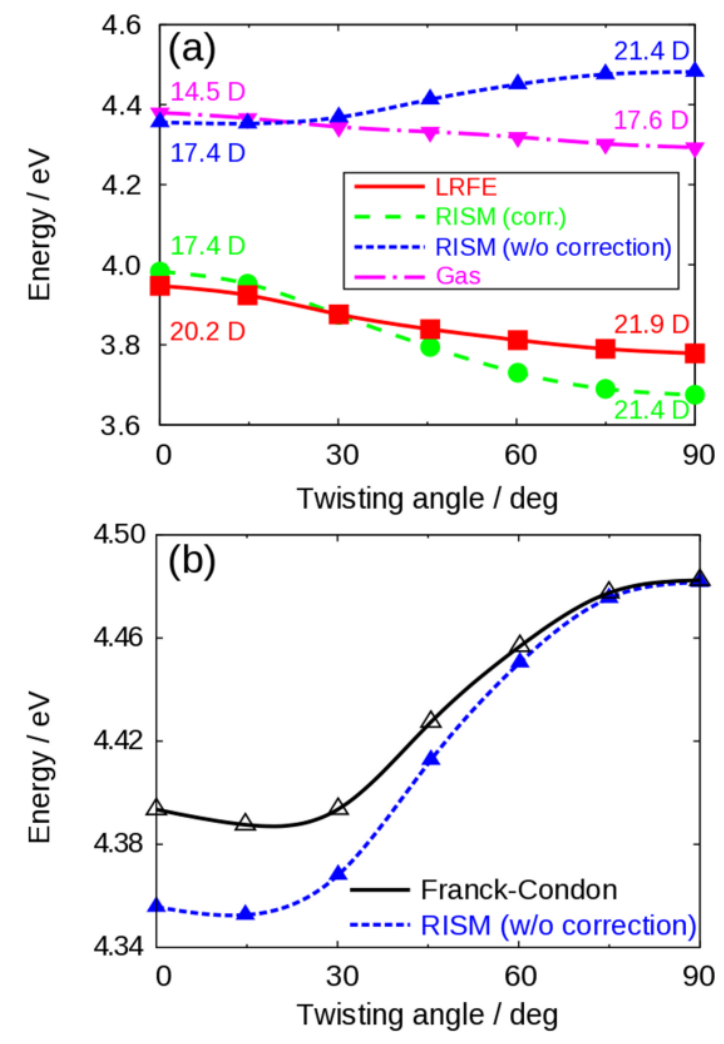

Figure 2. N. Minezawa 


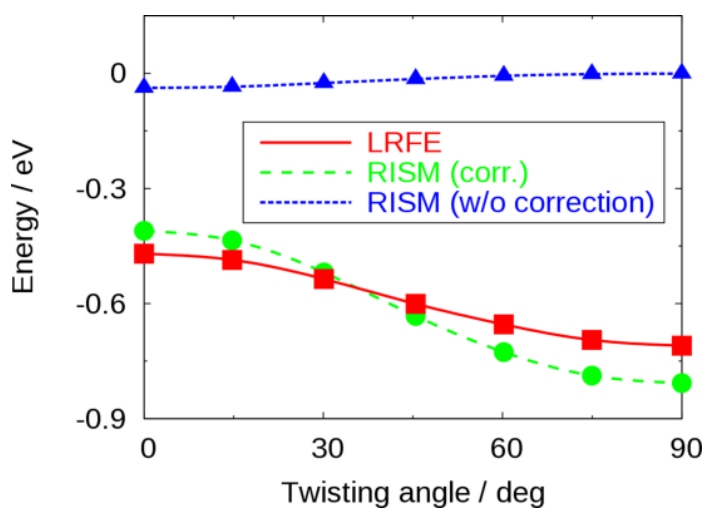

Figure 3. N. Minezawa 


\section{Table 1}

Absorption and emission energies of PBN at the (TD-)LC-BLYP/DH+(d) level. Values in parentheses are oscillator strengths, and experimental values are included in square brackets. ${ }^{\text {a }}$

\begin{tabular}{ccc}
\hline & Gas & Acetonitrile \\
\hline 2A & $4.72(0.625)[4.34]$ & \\
1B & $4.74(0.014)$ & $4.68(0.657)$ \\
2B & $4.80(0.001)$ & $4.74(0.012)$ \\
& Fluorescence & \\
LE & $4.26(0.712)[\sim 4.0]$ & $4.001)$ \\
TICT & $3.22(0.000)[3.56]$ & $2.41(0.000)[2.57]$
\end{tabular}

${ }^{a}$ See Ref. [36]. A weak shoulder around $310 \mathrm{~nm}(\sim 4.0 \mathrm{eV})$ is observed for the fluorescence spectrum measured in $n$-hexane and interpreted as the emission from the locally excited (LE) state. 


\section{References}

1. E. Runge, E. K. U. Gross, Phys. Rev. Lett. 52 (1984) 997.

2. M. E. Casida, in: D. P. Chong (Ed.), Recent Advances in Density-functional Methods, World Scientific, Singapore, 1995, p. 155.

3. F. Furche, R. Ahlrichs, J. Chem. Phys. 117 (2002) 7433.

4. J. G. Ángyán, Chem. Phys. Lett. 241 (1995) 51.

5. M. Cossi, V. Barone, J. Chem. Phys. 115 (2001) 4708.

6. R. Cammi, S. Corni, B. Mennucci, J. Tomasi, J. Chem. Phys. 122 (2005) 104513.

7. M. Caricato, B. Mennucci, J. Tomasi, F. Ingrosso, R. Cammi, S. Corni, G. Scalmani, J. Chem. Phys. 124 (2006) 124520.

8. R. Improta, V. Barone, G. Scalmani, M. J. Frisch, J. Chem. Phys. 125 (2006) 054103.

9. L. V. Slipchenko, J. Phys. Chem. A 114 (2010) 8824.

10. K. Sneskov, T. Schwabe, O. Christiansen, J. Kongsted, Phys. Chem. Chem. Phys. 13 (2011) 18551.

11. M. Caricato, J. Chem. Phys. 139 (2013) 044116.

12. A. Pedone, J. Chem. Theory Comput. 9 (2013) 4087. 
13. N. Minezawa, J. Chem. Phys. 138 (2013) 244101.

14. S. Ten-no, F. Hirata, S. Kato, J. Chem. Phys. 100 (1994) 7443.

15. H. Sato, F. Hirata, S. Kato, J. Chem. Phys. 105 (1996) 1546.

16. H. Sato, in: F. Hirata (Ed.), Molecular Theory of Solvation, Kluwer Academic, Dordrecht, 2003, pp. 61-99.

17. H. Sato, Phys. Chem. Chem. Phys. 15 (2013) 7450.

18. S. Gusarov, T. Ziegler, A. Kovalenko, J. Phys. Chem. 110 (2006) 6083.

19. D. Casanova, S. Gusarov, A. Kovalenko, T. Ziegler, J. Chem. Theory Comput. 3 (2007) 458.

20. D. Yokogawa, H. Sato, S. Sakaki, J. Chem. Phys. 126 (2007) 244504.

21. J. W. Kaminski, S. Gusarov, T. A. Wesolowski, A. Kovalenko, J. Phys. Chem. A 114 (2010) 6082.

22. M. J. F. Fernandez, H. Sato, Theor. Chem. Acc. 130 (2011) 299.

23. S. Hayaki, Y. Kimura, H. Sato, J. Phys. Chem. B 117 (2013) 6759.

24. S. Yamazaki, S. Kato, J. Chem. Phys. 123 (2005) 114510.

25. M. Higashi, S. Hayashi, S. Kato, J. Chem. Phys. 126 (2007) 144503.

26. T. Yamamoto, S. Kato, J. Chem. Phys. 126 (2007) 224514.

27. T. Mori, K. Nakano, S. Kato, J. Chem. Phys. 133 (2010) 064107. 
28. S. Aono, N. Minezawa, S. Kato, Chem. Phys. Lett. 492 (2010) 193.

29. R. M. Levy, M. Belhadj, D. B. Kitchen, J. Chem. Phys. 95 (1991) 3627.

30. K. Naka, A. Morita, S. Kato, J. Chem. Phys. 110 (1999) 3484.

31. W. Kohn, L. J. Sham, Phys. Rev. 140 (1965) A1133.

32. A. Morita and S. Kato, J. Am. Chem. Soc. 119 (1997) 4201.

33. Z. R. Grabowski, K. Rotkiewicz, W. Rettig, Chem. Rev. 103 (2003) 3899.

34. V. I. Tomin, K. Hubisz, Opt. Spectrosc. 110 (2011) 714.

35. L. Belau, Y. Haas, W. Rettig, Chem. Phys. Lett. 364 (2002) 157.

36. C. Cornelissen-Gude, W. Rettig, J. Phys. Chem. A 102 (1998) 7754.

37. A. B. J. Parusel, Phys. Chem. Chem. Phys. 2 (2000) 5545.

38. M. W. Schmidt, K. K. Baldridge, J. A. Boatz, S. T. Elbert, M. S. Gordon, J. H. Jensen, S. Koseki, N. Matsunaga, K. A. Nguyen, S. J. Su, T. L. Windus, M. Dupuis, J. A. Montgomery, Jr., J. Comput. Chem. 14 (1993) 1347.

39. M. S. Gordon, M.W. Schmidt, in: C. E. Dykstra, G. Frenking, K. S. Kim, G. E. Scuseria (Eds.), Theory and Applications of Computational Chemistry: The First Forty Years, Elsevier, Amsterdam, 2005, Chap. 41, pp. 1167-1189.

40. W. L. Jorgensen, J. M. Briggs, Mol. Phys. 63 (1988) 547. 
41. W. D. Cornell, P. Cieplak, C. I. Bayly, I. R. Gould, K. M. Merz, Jr., D. M. Ferguson, D. C. Spellmeyer, T. Fox, J. W. Caldwell, P. A. Kollman, J. Am. Chem. Soc. 117 (1995) 5179.

42. Y. Tawada, T. Tsuneda, S. Yanagisawa, T. Yanai, K. Hirao, J. Chem. Phys. 120 (2004) 8425.

43. M. Kamiya, H. Sekino, T. Tsuneda, K. Hirao, J. Chem. Phys. 122 (2005) 234111.

44. M. Chiba, T. Tsuneda, K. Hirao, J. Chem. Phys. 124 (2006) 144106.

45. M. Chiba, T. Tsuneda, K. Hirao, J. Chem. Phys. 126 (2007) 034504.

46. R. Baer, E. Livshits, U. Salzner, Annu. Rev. Phys. Chem. 61 (2010) 85.

47. T. H. Dunning, Jr., P. J. Hay, in: H. F. Schaefer III (Ed.), Methods of Electronic Structure Theory, Plenum, New York, 1977, p. 1.

48. M. J. G. Peach, P. Benfield, T. Helgaker, D. J. Tozer, J. Chem. Phys. 128 (2008) 044118. 